\title{
Association Between Perfluorinated Compounds and Pathological Conditions in Southern Sea Otters
}

\author{
Kurunthachalam Kannan ${ }^{\dagger, *}$, Emily Perrotta ${ }^{\dagger}$, and Nancy J. Thomas ${ }^{\ddagger}$ \\ ${ }^{\dagger}$ Wadsworth Center, New York State Department of Health and Department of \\ Environmental Health Sciences, School of Public Health, State University of New York at \\ Albany, Empire State Plaza, P.O. Box 509, Albany, New York 12201-0509, USA \\ ${ }^{\ddagger}$ US Geological Survey-Biological Resources Division, National Wildlife Health Center, \\ 6006 Schroeder Road, Madison, WI 53711-6223, USA
}

*Corresponding author: $\quad$ Tel: 518-474-0015; Fax: 518-473-2895; E-mail:

kkannan@wadsworth.org 


\section{Supplementary Information}

Figure S1. Box-and-whisker plots of PFOA and PFOS concentrations in sea otters stratified by nutritive condition. $\mathrm{EM}=$ emaciated; $\mathrm{EX}=$ excellent. An outlier for PFOS was removed in the diseased category, so $n=26$. Non-detects were assigned the LOQ value. Details of the plot can be found in the legend for Figure 3.
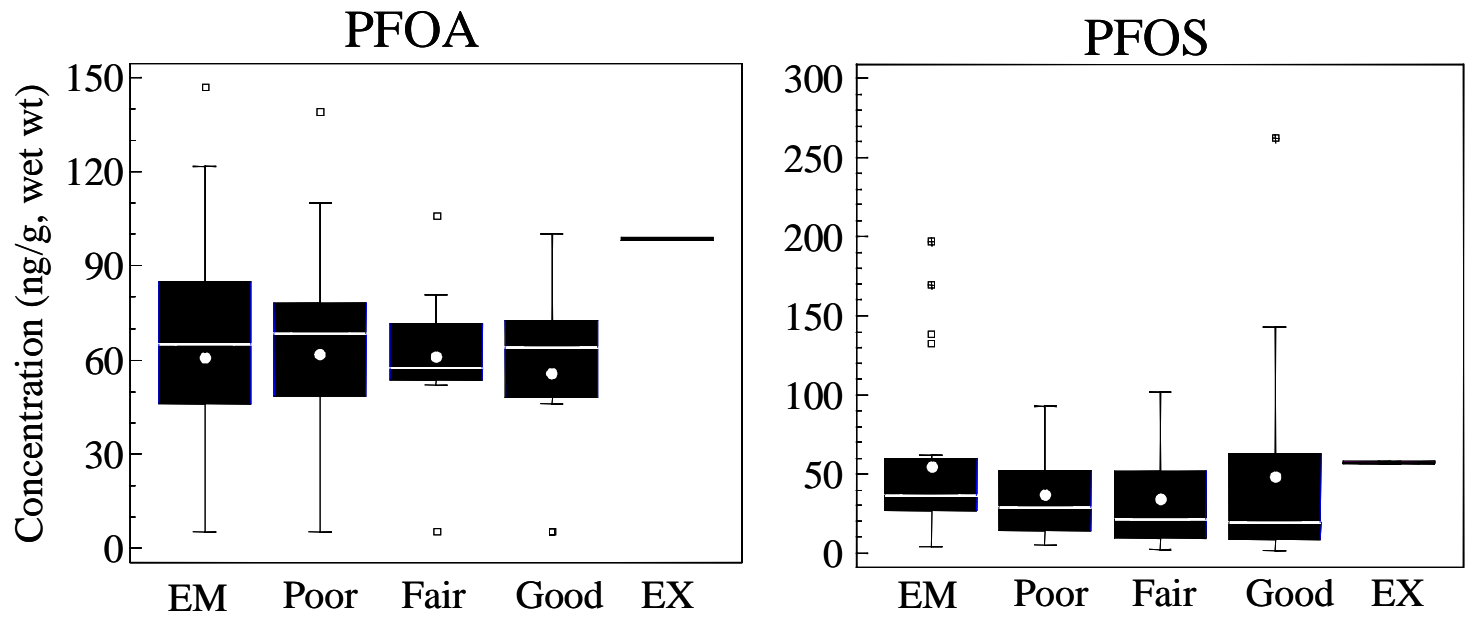
Figure S2. Box-and-whisker plots of PFOA and PFOS concentrations in livers of sea otters collected during high (spring and summer, $n=38$ ) and low (winter and fall, $n=42$ ) mortality seasons. An outlier for PFOS was removed in the diseased category. Nondetects were assigned the LOQ value. Details of the plot can be found in the legend for Figure 3.

Fig. S2

Kannan et al.
